Original Article

\title{
INFLUENCE OF BIOENHANCERS ON THE RELEASE PATTERN OF NIOSOMES CONTAINING METHOTREXATE
}

\section{Narayana Charyulu R., Gandhi Kinjal B., Jobin Jose, Sneh Priya, Shastry C.S.}

Department of Pharmaceutics, N.G.S.M. Institute of Pharmaceutical Sciences, Paneer, Deralakatte, Mangalore - 575018

Correspondence:

Narayana Charyulu R.

Vice Principal and HOD, Department of Pharmaceutics, Nitte Gulabi Shetty M emorial Institute of Pharmaceutical Sciences, Deralakatte, Mangalore-575018

Mobile : +919448164750 E-mail : charyulun@yahoo.co.in

\begin{abstract}
:
The aim of present study was to prepare sustained release formulations of niosomes of methotrexate (M TX) alone (N1 to N10) and along withbioenhancers (NB1 to NB9) by thin film hydration technique using span 60 as surfactant,cholesterol as membrane stabilizing agent, curcumin and piperine as bioenhancers and dicetyl phosphate (DCP) as charge inducing agent.All the formulations of niosomeswere characterized on the basis of physical appearance and entrapment efficiency. The invitro releasestudies of optimized formulation of niosomes of M TX alone and along with bioenhancers were performed and compared with pure drug released. The entrapment efficiency of M TX in optimized formulation of niosomes containing M TX along with bioenhancers was found to $56.9 \%$ and entrapment efficiency of bioenhancerscurcumin and piperinewas found to be $40.30 \%$ and $69.1 \%$ respectively. In vitro drug release of optimized formulationsof niosomes of M TX without and with bioenhancers (F3) was found to be $98.89 \%$ and $60.97 \%$ at the end of $12 \mathrm{~h}$ respectively. Results concluded that Niosomes of M TX containing bioenhancers followed sustain release pattern.
\end{abstract}

Keywords: Methotrexate, Thin Film Hydration Technique, Entrapment efficiency, Invitro study

\section{Introduction :}

Cancer still remains as one of the fatal disease inspite of outstanding improvements in molecular biology, genetics, and chemotherapy. Treatment of cancer involves the use of chemotherapy, radiation therapy, and surgery ${ }^{1,2,3}$. As antitumor agents have high potential to induce side effects and toxicity, localization of the drug to the tumor site would certainly optimize the therapy.The concept of targeted drug delivery is designed for attempting to concentrate drug in the tissues of interest and thereby reducing the relative concentration of medication in the remaining tissues ${ }^{4}$.Certain carriers like liposomes, niosomes, microsphers, nanopaticles, cellular carriers like erythrocytes and lymphocytes may be used to ferry the drug to the required site. Ideally, such carriers should be targeted the pathological area to provide the maximum therapeutic efficacy. Niosomes have gained increasing importance as a means of targeting of drugs. Niosomes have received attention for their potential as drug delivery vehicles due to advantages like higher flexibility, better bioavailability, increased efficacy, and therapeutic index ${ }^{5,6}$. Bioavailability of drug encapsulated in niosome can be enhanced by encapsulating the drug along with bioenhancers in the niosomal vesicles.The coadministration of bioenhancer like piperine with MTX inhibiting the P-glycoprotein and cytochrome p-450 enzymes enhances the efficacy of drug, makes drug more effective against cancer and transporter inhibitors like curcumin increases the intracellular drug accumulation and restores the chemosensitivity ${ }^{7,8}$.

MTX is an antimetabolite and antifolate drug.lt acts by inhibiting the metabolism of folic acid.M TX is a standard chemotherapeutic agent which exhibits a dose dependent toxicity. The most common problem encountered with MTX is the development of resistance to tumors. Relatively small increase in drug resistance in cancer cells is thus sufficient to render the drug ineffective. Hence there is a need to improve its acceptability by minimizing the intensity of side effects and thus increasing the therapeutic efficacy of the drug.

The aim of the present study was to utilize the principles of niosomal drug delivery systems to formulate a sustained release system for M TX alone and along with bioenhancers 
(a mixture of piperine and curcumin)by thin film hydration technique such that an increased entrapment with prolong the release of drug from niosones and also provided better stability to the formulation.

\section{Materials and Methods:}

\section{Materials:}

M TX was a gift sample from Khandelwal laboratories Pvt, Ltd. (M umbai, India). Span 60(surfactant) was obtained from LobachemiePvt. Ltd. (Mumbai, India). M ethanol, hydrochloric acid and chloroform were obtained from Merck India Ltd, (Mumbai, India). Cholesterol and potassium dihydrogen phosphate were obtained from HiM edia Laboratories Pvt, Ltd. (M umbai, India). Curcumin extract and piperine extracts were obtained from Green Grover's Pvt Ltd. (Bangalore, India). DCPwas obtained from Sigma Aldrich Chemicals, (Bangalore, India). Sodium chloride, sodium hydroxide and disodium hydrogen phosphate were obtained fromCDH Laboratory Ltd. (Delhi, India). All chemicals used were of analytical grade

\section{Methods:}

\section{Preparation of Niosomes of MTX alone and along with Bioenhancers}

Multilamellar vesicles of MTX alone and along with bioenhancers were prepared by thin film hydration technique using rotary flash evaporator as described method of Bangham, reported by Juliano and Daoud ${ }^{9}$. Accurately weighed quantity of cholesterol, span $60^{10,11}$ and DCPwere dissolved in minimum quantity (about $3 \mathrm{ml}$ ) of a mixture of chloroform: methanol (2:1) in a $250 \mathrm{ml}$ round bottom flask. Round bottom flask was then attached to a rotary evaporator.The organic solvent mixture was evaporated in a rotary flash evaporator under a vacuum of 25 inches of $\mathrm{Hg}$ at $60 \pm 2^{\circ} \mathrm{C}$ and the flask rotated at $100 \mathrm{rpm}$ until a very thin, smooth and dry film of surfactant was formed on the inner surface of the flask, The dry lipid film was slowly hydrated with $5 \mathrm{ml}$ phosphate buffer saline (PBS) of pH 7.4 containing $10 \mathrm{mg} \mathrm{M} \mathrm{TX} \mathrm{drug} \mathrm{alone} \mathrm{and} \mathrm{with}$ $10 \mathrm{ml}$ PBS pH 7.4 containing $10 \mathrm{mg} \mathrm{MTX}$ drug and accurate quantity of bioenhancers at a temperature of $60 \pm 2 \stackrel{\circ}{ } \mathrm{C}$ for a period of $1 \mathrm{~h}$. It formed homogenous suspension of multilamellar vesicles (MLVs).TheMLVs suspension was sonicated to form small unilamellar vesicles (SUVs)of niosomes by using probe sonicator. The final niosomal suspension was further hydrated at $4^{\circ} \mathrm{C}$ for overnightto stabilize the formulation. The amount of span,cholesterol and bioenhancers to be loaded was selected on the basis of entrapment efficiency of the vesicles.The compositions of different formulation of niosomes are given in Table 1a and lb.

\section{Characterization of Niosomes of MTX alone and along with Bioenhancers}

\subsection{Entrapment efficiency}

For determination of entrapment efficiency, unentrapped drug in the niosomal formulation was seperated using centrifugation at 20,000 rpm for $1 \mathrm{~h}$ at $4 \stackrel{\circ}{ } \mathrm{C}$. The supernatant contains unentrapped M TX was removed and the remaining pellet in the centrifuge tube resuspended in $0.1 \mathrm{~N}$ sodium hydroxide (as MTX is highly soluble in $0.1 \mathrm{~N}$ $\mathrm{NaOH}$ ) and vortexed thoroughly for 3 min. After vortexing $1 \mathrm{ml}$ of the suspension was taken in a micropipette and transferred to a test tube. To this added $5 \mathrm{ml}$ of methanol and was further vortexed for 2 min.The absorbance of resulting solution was measured using a shimadzu UV Spectrophotometer(1700) at $292 \mathrm{~nm}$ after suitable dilution with methano ${ }^{10}$.

\subsection{In Vitro release study of optimized formulation}

A volume of $1 \mathrm{ml}$ niosomal dispersion (encapsulation efficiency: $56.9 \%$ ) was put in a dialysis bag (M WCO 12,000 Da,Sigma-Aldrich, USA.). The dialysis bag was suspended in $300 \mathrm{ml}$ phosphate buffer $\mathrm{pH} 7.4$ and maintained at $37 \pm 0.2^{\circ} \mathrm{C}$. The medium was stirred continuously during the release study. At predetermined time intervals of $15 \mathrm{~min}, 30$ $\min , 45 \mathrm{~min}, 60 \mathrm{~min}, 2,3,4,5,6,7,8,9,10,11$ and $12 \mathrm{~h}, 5 \mathrm{ml}$, aliquots were sampled and replaced with $5 \mathrm{ml}$ fresh phosphate buffer $\mathrm{pH} \mathrm{7.4.} \mathrm{The} \mathrm{concentration} \mathrm{of} \mathrm{M} \mathrm{TX} \mathrm{was}$ determined by the UV spectrophotometer (Shimadzu UV $1700)$ at $303 \mathrm{~nm}^{11,12}$.

\section{Result and Discussion :}

Niosomes of M TX without and with bioenhancers such as curcumin and piperine were prepared by thin film hydration method by using span 60 and cholesterolas a 
surfactant. They are optimized on the basis of observation and maximum percentage drug entrapment (PDE).Entrapment efficiency

Theoptimization ofniosomes ofM TX without bioenhancers depends on the basis of entrapment efficiency. The entrapment efficiency was varied as concentration of surfactant varied. The amount of span 60 was increased by keeping drug and cholesterol concentration constant. As the amount of span 60 increased, the PDE of drug was also increased upto the formulation N6 (1:15 ratioof drug and span60) and further increasing the amount of span $60 \mathrm{did}$ not change encapsulation efficiency. The higher entrapment of span 60 may be due to their high phase transition temperature and hydrophobic in nature.

The amount of cholesterol was increased by keeping drug and surfactant ratio constant. The ratio1:15:2 gave highest encapsulation efficiency due to stabilizing effect of cholesterol. The cholesterol in niosomes greatly affects the membrane properties of the bilayers by reducing the rotational freedom of hydrocarbon chains. Cholesterol also eliminates the gel to liquid phase transition of the vesicle bilayers and induces permanent transition of the gel-state bilayer to an ordered liquid crystalline state. Both these mechanisms make the bilayers more stable leading to increase in entrapment efficiency. Further increase in cholesterol concentration, decreased the fluidity of the bilayers by filling empty spaces among the surfactant molecules and results of the membrane become more rigid and ultimately decreased the encapsulation efficiency (Table 2a).

Optimization of niosomes of M TX with bioenhancers was done on the basis of entrapment efficiency. The formulation in which bioenhancers were added $40 \mathrm{mg}$ of curcuminand $10 \mathrm{mg}$ of piperine) produced a uniform dispersion with lower drug entrapment. Further increase in curcumin concentration $(50 \mathrm{mg})$ by keeping piperine concentration constant (10mg) produced a uniform suspension with an acceptable PDE. Further increase in concentration of piperine $(>10 \mathrm{mg})$ reduce the entrapment efficiency. So the formulation NB6 containing curcumin (50mg) and piperine (10 mg)as a bioenhancers was found to be an optimized formulation which gave highest drug entrapment (55.1 $\pm 0.49 \%$ ) (Table 2b).

\section{In Vitro Release Study :}

The invitro release study revealed that the release of the drug was sustained on encapsulation in niosomes. The free drug released approximately $98.77 \%$ of the drug within 60 minwhereas the same percentage of drug release from niosomes of M TXwas occurring at the end of $11 \mathrm{~h}$. Release of MTX from niosomes was biphasic with an initial faster release for $3 \mathrm{~h}$ followed by a period of slow release. Thus, the study revealed that initially there was a high rate of drug release, which may be due to the release of the adsorbed drug from the lipophilicregion of niosomes, which help to achieve the optimum loading dose.The drug diffuses slowly after $3 \mathrm{~h}$ due to the presence of cholesterol in the formulation which affects the fluidityby making it more rigid. As the amount cholesterol increased, they filled the pores of vesicular bilayers and abolished the gel-liquid phase transition of the niosomal systems. This confirms that addition of cholesterol acted as a membrane stabilizing agent that decreased the permeability and helped to sustain the release.

The maximum release of drug from niosomes containing M TX along with bioenhancers was $60.9 \%$ at the end of $12 \mathrm{~h}$. The reason for slower release of the drug from niosomes encapsulated complex may be the interaction of complex with the lipid/surfactant bilayers and bioenhancers.These results indicate that the release of M TX followed a sustain release pattern (Figure: 1 ).

\section{Conclusion:}

The use of various pharmaceutical nanocarriers has become one of the most important areas of nanomedicine. Niosomes of methotrexate alone and along with bioenhancers such as curcumin and piperine were prepared by thin film hydration method by using surfactant span 60 and cholesterol that were optimized on the basis of entrapment efficiency. The in vitrostudy revealed that the release pattern of the drug was sustained in niosomes and it was further significantly sustained by addition of bioenhancers. Further in vivo and stability studies can be 
performed to see the pharmacological activity as well as the stability of the formulation because the stability of niosomes is a great issue and a major challenge in commercializing the formulations.

\section{Acknowldegements:}

The authors are expressing their sincere gratitude to the Nitte University for providing necessary facilities to carry out this research work.

Table 1a: Composition of different formulation of niosomes of M TX without bioenhancers

\begin{tabular}{|l|c|c|c|c|c|c|}
\hline Batch name & $\begin{array}{c}\text { MTX } \\
(\mathbf{m g})\end{array}$ & $\begin{array}{c}\text { Span 60 } \\
(\mathbf{m g})\end{array}$ & $\begin{array}{c}\text { Cholesterol } \\
(\mathbf{m g})\end{array}$ & $\begin{array}{c}\text { DCP } \\
(\boldsymbol{\mu m o l})\end{array}$ & $\begin{array}{c}\text { Organic } \\
\text { solvent }(\mathbf{m l})\end{array}$ & $\begin{array}{c}\text { Hydration } \\
\text { Volume (ml) }\end{array}$ \\
\hline N1 & s10 & 50 & 10 & 7 & 3 & 5 \\
\hline N2 & 10 & 75 & 10 & 7 & 3 & 5 \\
\hline N3 & 10 & 100 & 10 & 7 & 3 & 5 \\
\hline N4 & 10 & 125 & 10 & 7 & 3 & 5 \\
\hline N5 & 10 & 150 & 10 & 7 & 3 & 5 \\
\hline N6 & 10 & 175 & 10 & 7 & 3 & 5 \\
\hline N7 & 10 & 150 & 20 & 7 & 3 & 5 \\
\hline N8 & 10 & 150 & 30 & 7 & 3 & 5 \\
\hline N9 & 10 & 150 & 40 & 7 & 3 & 5 \\
\hline N10 & 10 & 150 & 50 & 7 & 3 & 5 \\
\hline
\end{tabular}

Table 1b: Composition of different formulation of niosomes of M TX along with bioenhancers

\begin{tabular}{|l|c|c|c|c|c|c|c|c|}
\hline $\begin{array}{l}\text { Batch } \\
\text { name }\end{array}$ & $\begin{array}{c}\text { MTX } \\
(\mathbf{m g})\end{array}$ & $\begin{array}{c}\text { Span } \\
\mathbf{6 0}(\mathbf{m g})\end{array}$ & $\begin{array}{c}\text { Cholesterol } \\
\mathbf{( m g )}\end{array}$ & $\begin{array}{c}\text { Curcumin } \\
\mathbf{( m g )}\end{array}$ & $\begin{array}{c}\text { Piperine } \\
\mathbf{( m g )}\end{array}$ & $\begin{array}{c}\text { DCP } \\
(\boldsymbol{\mu m o l})\end{array}$ & $\begin{array}{c}\text { Organic } \\
\text { solvent (ml) }\end{array}$ & $\begin{array}{c}\text { Hydration } \\
\text { Volume (m) })\end{array}$ \\
\hline Nb1 & 10 & 150 & 20 & 5 & 10 & 7 & 3 & 10 \\
\hline Nb2 & 10 & 150 & 20 & 10 & 10 & 7 & 3 & 10 \\
\hline Nb3 & 10 & 150 & 20 & 20 & 10 & 7 & 3 & 10 \\
\hline NB4 & 10 & 150 & 20 & 30 & 10 & 7 & 3 & 10 \\
\hline NB5 & 10 & 150 & 20 & 40 & 10 & 7 & 3 & 10 \\
\hline NB6 & 10 & 150 & 20 & 50 & 10 & 7 & 3 & 10 \\
\hline NB7 & 10 & 150 & 20 & 50 & 20 & 7 & 3 & 10 \\
\hline NB8 & 10 & 150 & 20 & 50 & 30 & 7 & 3 & 10 \\
\hline NB9 & 10 & 150 & 20 & 50 & 40 & 7 & 3 & 10 \\
\hline
\end{tabular}

Table 2a.Optimization of niosomes of M TX withoutbioenhancers

\begin{tabular}{|c|c|c|}
\hline Batch name & Observation & \% Drug entrapped* \\
\hline N1 & Flaking & $22.17 \pm 1.667$ \\
\hline N2 & Flaking & $29.89 \pm 1.025$ \\
\hline N3 & Flaking & $38.49 \pm 1.351$ \\
\hline N4 & Flaking & $43.35 \pm 0.920$ \\
\hline N5 & Uniform dispersion & $50.73 \pm 0.714$ \\
\hline N6 & Non-uniform dispersion & $21.35 \pm 0.840$ \\
\hline N7 & Uniform dispersion without flaking & $\mathbf{5 6 . 9 \pm 1 . 3 3 1}$ \\
\hline N8 & Uniform dispersion lower PDE & $53.85 \pm 0.818$ \\
\hline N9 & Uniform dispersion Lower PDE & $51.03 \pm 0.512$ \\
\hline N10 & Uniform dispersion lower PDE & $49.48 \pm 0.918$ \\
\hline
\end{tabular}

* Data are expressed as Mean \pm SD. SD =Standard Deviation 
Table $\mathbf{2 b}$ :Optimization of niosomes of MTX with bioenhancers

\begin{tabular}{|l|l|l|l|l|}
\hline Batch & Observation & \% Drug & \%Curcumin & \% Piperine \\
\hline name & & entrapped* & entrapped* & entrapped* \\
\hline NB1 & Flaking & $18.90 \pm 0.21$ & $27.66 \pm 0.66$ & $10.05 \pm 0.36$ \\
\hline NB2 & Flaking & $23.46 \pm 0.39$ & $31.66 \pm 0.67$ & $23.58 \pm 0.21$ \\
\hline NB3 & Flaking & $25.89 \pm 0.44$ & $32.78 \pm 0.12$ & $24.30 \pm 0.64$ \\
\hline NB4 & Uniform dispersion & $27.67 \pm 0.62$ & $35.94 \pm 0.51$ & $32.10 \pm 0.26$ \\
\hline NB5 & Uniform Dispersion & $32.24 \pm 0.51$ & $39.01 \pm 0.21$ & $39.00 \pm 0.82$ \\
\hline NB6 & Uniform Dispersion & $\mathbf{5 5 . 1} \pm \mathbf{0 . 4 9}$ & $\mathbf{4 0 . 3 0} \pm \mathbf{0 . 6 7}$ & $\mathbf{6 4 . 3 1 \pm 0 . 9 6}$ \\
\hline NB7 & Non-uniform Dispersion & $49.89 \pm 0.53$ & $33.87 \pm 0.54$ & $29.00 \pm 0.38$ \\
\hline NB8 & Non-uniform Dispersion & $47.77 \pm 0.55$ & $31.33 \pm 0.86$ & $27.13 \pm 0.27$ \\
\hline NB9 & Non-Uniform Dispersion & $46.98 \pm 0.34$ & $28.00 \pm 0.96$ & $26.18 \pm 0.91$ \\
\hline
\end{tabular}

* Data are expressed as M ean \pm SD. SD $=$ Standard Deviation

Figure: 1 In vitro release of pure drug, niosomes of M TXalone and niosomes of M TX along with bioenhancers

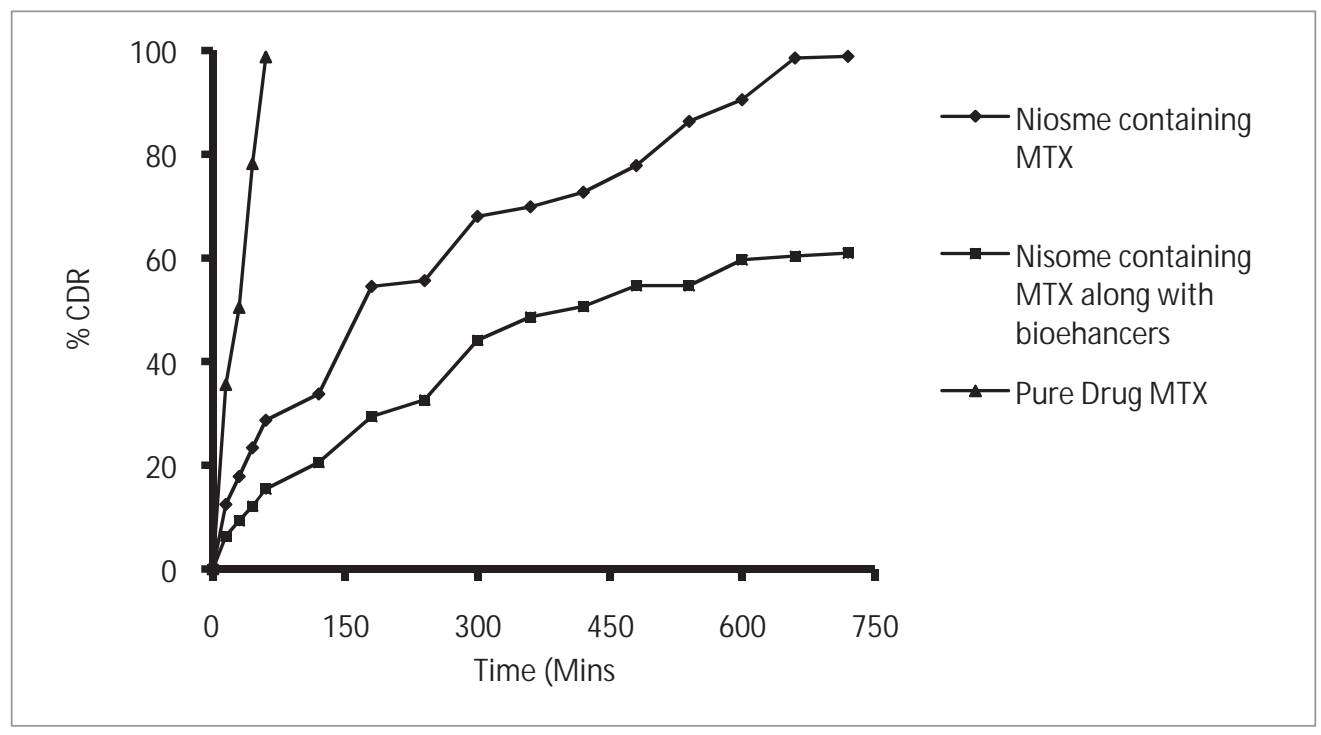

\section{References:}

1. Aggarwal B B, Kumar A, Bharti A C. Anticancer potential of curcumin: Preclinical and clinical studies. Anticancer Res. 2003; 23(1A): 363 - 98.

2. Harsh Mohan. "Neoplasia" in textbook of pathology, 2005;P:142, Jaypee Brothers, New Delhi.

3. Harvey Ladies et al. "Cancer" Molecular cell biology, 2005; P:1267, Scientific American book, London.

4. M. Reza Mozafari. Nanocarrier Technologies: Frontiers of Nanotherapy. 2006; P: 1-16, Springer, Netherlands.

5. Torchillin VP. Multifunctional nanocarriers. Adv drug del rev. 2006; 58(14): $1533-9$

6. Torchillin $\vee \mathrm{P}$. Targeted pharmaceutical nanocarriers for cancer therapy and imaging. The AAPS J ournal. 2007; 9 (2): E128-47.

7. Gottesman M.M. M echanisms of cancer drug resistance. Annual rev. med. 2002; (53):615-27.

8. Zhang S, X, M orris M E. Flavanoids are inhibitors of breast cancer resistance protein (ABCG2)- Mediated transport.Mole pharmaco. 2004:65(5):1208-16.

9. Joshi M, Misra A. Dry powder inhalation of liposomal ketotifen fumarate: Formulation and characterization. Int. J. Pharm. 2001;223(1-2): 15-27.

10. Singh C H, Jain C P, Kumar B N. Formulation, characterization, stability and in vitro evaluation of nimesulide niosomes. Pharmacophore. 2011; 2 (3): 168-85.

11. Karki R, Mamatha G C, Subramanya G, Udupa N. Preparation, characterization and tissue disposition of niosomes containing isoniazid. Rasayan J. Chem. 2008;1(2):224-7.

12. Yang Z J, Huang W H, Wong Y.F, Zhao Z Z, Liu L. Development of liposomal salbutamol sulphate dry powder inhaler formulation. Biol. Pharm. Bull. 2010; 33(3): 512-7. 